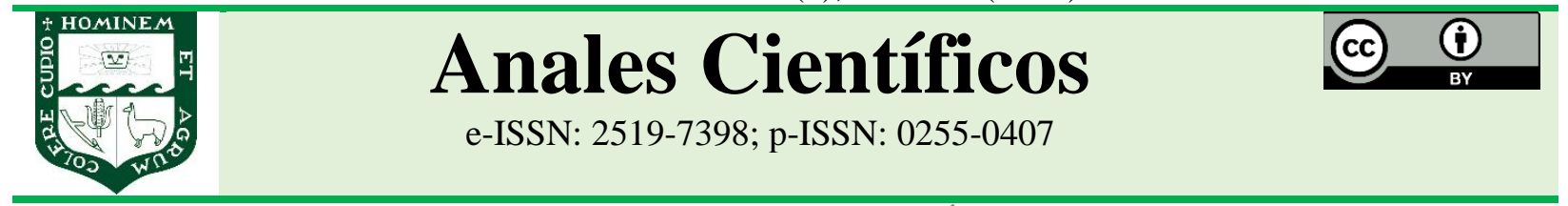

ARTÍCULO ORIGINAL - RESEARCH ARTICLE

http://dx.doi.org/10.21704/ac.v82i2.1793

\title{
EVALUACIÓN DE LA VIABILIDAD DE SEMILLAS DE SACHA INCHI Plukenetia volubilis L. Conservadas a $4^{\circ} \mathrm{C}$.
}

\section{Evaluation of the viability of sedes of sacha inchi Plukenetia volubilis L. preserved at $4^{\circ} \mathrm{C}$}

\author{
Fredesvinda Carrillo ${ }^{1 *}$ (D); Ana Laura Rucabado ${ }^{2}$ (D) \\ ${ }^{1}$ Instituto Nacional de Innovación Agraria. Lima, Perú. \\ * E-mail: fcarrillo@inia.gob.pe
}

Recibido: 02/10/2020; Aceptado: 04/10/21; Publicado: 31/12/2021

\begin{abstract}
Sacha inchi Plukenetia voluvilis L., is a species native to and promising cultivation in the Peruvian Amazon, which has nutritional properties (omega 3 ) beneficial for the human body=, therefore the interés of studying and evaluating the shelf life under partular conditions. The aim of this study was to evaluate the viability of sacha inchi seeds, stored in a cold chamber, with moisture contents between 4.0 and 6.8\%, for eigth years. 27 accessions of Sacha inchi were used, the seed of which were partially and completely scarified, and were soaked in distilled water for 12 hours, then disinfected with 3\% captan (fungicide). The scarified seeds of a total of 27 accesiion were sown on towel paper moistened with distilled water and placed in the germinator under controlled light and temperature conditions, while theseed of the partially scarified accessions were sown on fattening trays containing a misxture of sand, moss and coconut fibre, a placed in a tinglage to the environment. The results show that the germination process on scarfied seeds located in the germinator begins after 12 days plant and 20 days in partially scarified seeds placed in the tingled. The viability of the seeds after 8 years of cold storage was $80-100 \%$ in $44 \%$ of the material evaluated. In addition, sacha inchi was found to have orthodox behaviour and can be kept with moisture content of the seed between 4,0 to $7.5 \%$.
\end{abstract}

Keywords. sacha inchi | orthodox | viability | conservation | accesiones

\section{RESUMEN}

El sacha inchi Plukenetia voluvilis L., es una especie nativa y cultivo promisorio de la Amazonia peruana, que tiene propiedades nutricionales (omega 3 ) benéficos para el organismo humano, por ello el interés de estudiar y evaluar el tiempo de conservación bajo condiciones particulares. El objetivo del presente trabajo, fue evaluar la viabilidad de las semillas de sacha Inchi=conservadas en cámara fría, con contenidos de humedad entre 4.0 a 7.5\%, durante ocho años de almacenamiento. Se emplearon 27 accesiones de sacha inchi, cuyas semillas fueron escarificadas en forma parcial y total, y se remojaron en agua destilada por 12 horas, luego fueron desinfectadas con captan al 3\% (fungicida). Las semillas escarificadas en forma total de 27 accesiones se sembraron sobre papel toalla humedecido con agua destilada y ubicadas en la germinadora bajo condiciones controladas de luz y temperatura, mientras que las semillas de las accesiones escarificadas parcialmente se sembraron en bandejas para almácigos las cuales contenianuna mezcla de arena, musgo y fibra de coco, y ubicadas en un tinglado al medio ambiente. Los resultados muestran que el proceso de germinación en semillas escarificadas ubicadas en la germinadora se inicia a los 12 días 
y 20 días en las semillas parcialmente escarificadas y ubicadas en el tinglado. La viabilidad de las semillas después de ocho años de conservación en cámara fría, fue de $80-100 \%$ en el $44 \%$ del material evaluado. Además, se comprobó que sacha inchi tiene comportamiento ortodoxo, y se puede conservar con contenido de humedad entre 4,0 a $7,5 \%$.

Palabras clave: sacha inchi | accesiones | ortodoxa | viabilidad | conservación | cámara fría

Forma de citar el artículo (Formato APA):

Carrillo, F., \& Rucabado, A. (2021). Evaluación de la viabilidad de semillas de sacha inchi Plukenetia volubilis L. conservadas a $4^{\circ} \mathrm{C}$. Anales Científicos. 81(2), 303-311. http://dx.doi.org/10.21704/ac.v82i2.1793

Autor de correspondencia (*): Fredesvinda Carrillo. Email: fcarrillo@inia.gob.pe

(C) Los autores. Publicado por la Universidad Nacional Agraria La Molina.

This is an open access article under the CC BY

\section{INTRODUCCIÓN}

El Sacha Inchi, Plukenetia volubilis L., es una especie de la familia Euphorbiacea, se distribuye desde América Central hasta Bolivia (Brack, 1999). Es un cultivo nativo de la Amazonía peruana que crece en estado silvestre y cultivado (Brack, 2005). En el Perú se le encuentra en Amazonas, Huánuco, Madre de Dios, San Martín Ucayali y Loreto, (Alcívar (2014). Fue sembrado desde la época prehispánica en la costa peruana, habiéndose encontrado semillas y representaciones en cerámica (Brack, 2003). Su nombre deriva de las palabras quechuas "sacha", que significa silvestre e "inchi", por el maní que produce. Se conoce también como sacha yuchi, sacha yuchiqui, sacha inchik, maní del monte, maní silvestre, maní del Inca (Alayon \& Echeverry, 2016).

La propagación de sacha inchi es a través de la semilla, pero se puede hacer por injerto para solucionar problemas fitosanitarios; por lo que antes de sembrar se deben desinfectar las semillas, para evitar enfermedades fungosas. (Dosert, et al.; 2009). Asimismo, las semillas poseen propiedades nutricionales, son importantes en la industria del aceite a nivel nacional e internacional. $\mathrm{La}$ semilla contiene un aceite rico en antioxidantes y ácidos grasos poliinsaturados, excelente para las industrias alimentarias y animales, así como en la cosmética (Alayon \& Echeverry, 2016). García (2015), refiere que las semillas de sacha inchi también contiene proteínas y vitamina $\mathrm{E}$ (tocoferoles y tocotrifenoles), comparados con otras semillas de oleaginosas. Manco (2007), citada por Flores (2010), resalta que la semillas de sacha Inchi posee hasta $33 \%$ de contenido de proteínas. No existen muchos estudios referidos a los efectos del almacenamiento sobre la viabilidad de la semilla de sacha inchi conservada en cámara fría. Manco (2008), citado por Antoniolli \& Arfini (2013), refiere que las semillas de sacha inchi pierden su viabilidad (60\%), por la presencia de aceites, por ello no se deben almacenar más de 60 días. Menges et al (2014) citado por Mancipe (2018), menciona que la viabilidad de la semilla es una medida de calidad, importante en el monitoreo periódico de semillas que se encuentran bajo almacenamiento, con el fin de asegurar el éxito de un programa de conservación ex situ.

El potencial fisiológico de las semillas depende de las condiciones de almacenamiento, para mantener la viabilidad de las semillas por períodos prolongados de tiempo (Navarro \& Lescano, 2008). El proceso de deterioro se refiere a la calidad de la semilla, y su conservación depende de la temperatura y la humedad de almacenamiento. (Aramendiz, et al., 2007). No todas las especies tienen los mismos requerimientos para la germinación. Las semillas muestran una gran variedad de respuestas, frente a las condiciones a las cuales están expuestas, que pueden considerarse como adaptaciones para maximizar la supervivencia en un ambiente impredecible (Faife \& Toledo, 2007). Especies de vida corta, puede aumentar su longevidad por un adecuado almacenamiento, las especies de vida intermedia pueden permanecer viables entre 2 a 15 años, bajando su contenido de humedad y a baja temperatura (Sánchez et al., 2011).

Los ensayos de germinación son los procedimientos más comunes para evaluar la calidad fisiológica de un lote de semillas. (García et al., 2016). Mientras que Lazos et al. (2015) refieren que las semillas difieren en su tolerancia a la desecación que sigue tras su 
diseminación. Las semillas se clasifican en ortodoxas, recalcitrantes e intermedias, las primeras toleran deshidratación o secado, incrementando su longevidad y permanecen viables. sin embargo, las recalcitrantes no toleran deshidratación, y las intermedias se pueden secar, pero a no muy bajo contenido de humedad (Doria, 2010).

En relación a sacha inchi, no se dispone o existe poca literatura referida a la conservación de semillas en bancos de germoplasma y los efectos en la calidad y longevidad de las mismas, como hay en otras especies. Los bancos de germoplasma tienen como objetivo general la conservación a largo plazo y la accesibilidad del germoplasma vegetal, es una estrategia muy importante sobre todo en especies amenazadas. (Hernández - Coronado et al., 2018).

El presente trabajo tiene por objetivo evaluar la viabilidad de las semillas de sacha inchi, conservadas durante ocho años de almacenamiento en cámara fría $\left(2{ }^{\circ} \mathrm{C}\right.$ a $\left.4{ }^{\circ} \mathrm{C}\right)$, con contenidos de humedad entre 4.0 a $7.5 \%$. En la investigación se empleó sustratos y condiciones ambientales diferentes, además del tipo de escarificado que pueden influir en la viabilidad de las semillas de sacha inchi.

\section{MATERIALES Y MÉTODOS}

El trabajo se realizó en el laboratorio del Banco de Semillas de la Subdirección de Recursos Genéticos, de la Dirección de Recursos Genéticos y Biotecnología DRGB, ubicado en la Sede Central del INIA, distrito La Molina, provincia de Lima, Perú. Localizado a una altitud de 248 msnm, latitud -12.01 y longitud -17.03.

Se emplearon semillas de 27 accesiones de sacha inchi (Plukenetia volubilis L.) de la campaña de siembra del año 2011, provenientes de la Colección de Sacha Inchi, establecida en la Estación Experimental Agraria El Porvenir -Tarapoto. Este material fue previamente secado para bajar su contenido de humedad y luego almacenado en sobres de poliéster laminado herméticamente sellado, y conservado en el Banco de Semillas a temperatura controlada que oscila entre $2^{\circ} \mathrm{C}$ a $4{ }^{\circ} \mathrm{C}$. El tiempo de almacenamiento de las semillas al año 2019 fue de ocho años, el contenido de humedad de las semillas varió entre $4,0 \%$ y $7,5 \%$.
Para la determinación de contenido de humedad se empleó el método del horno a bajas temperaturas (101$103{ }^{\circ} \mathrm{C}$ ), las semillas fueron trituradas previamente con ayuda de un molino de semillas y de la muestra molida se pesó 3 repeticiones de aproximadamente de $0.5 \mathrm{~g}$ cada una, utilizando recipientes de aluminio con tapa, las cuales se colocaron en el horno a la temperatura indicada por espacio de 17 horas (peso inicial), luego del tiempo establecido, los recipientes se retiran del horno y se disponen en un desecador y se espera a que enfríe por 45 minutos para volver a pesar ( peso final) y por diferencia de pesos se obtiene el $\%$ de humedad de la muestra, teniendo en cuenta que entre los resultados no haya diferencia de más de $0.2 \mathrm{~g}$.

Los sobres conteniendo las semillas se retiraron de la cámara fría, luego se extrajeron las semillas y se colocaron en sobres de papel, en número de 18 a 21 semillas. A continuación fueron escarificadas parcialmente y en forma total con ayuda de un alicate, para romper la cubierta. Las semillas escarificadas parcialmente, así como las escarificadas totalmente, se remojan por 12 horas en agua destilada. Después, fueron desinfectadas por cinco minutos, colocándolas en un vaso de precipitación de $250 \mathrm{ml}$, al cual se agregó fungicida Captan al 3\% cubriendo las semillas, luego se enjuagaron tres veces con agua destilada.

Una vez desinfectadas las semillas completamente escarificadas se distribuyeron en forma uniforme $\mathrm{y}$ distanciadas, sobre papel toalla (esterilizado), previamente rotulado con el número de accesión, fecha y número de semillas, luego se humedeció con agua destilada y se cubrió con otro papel toalla humedecido, seguidamente se enrollo suavemente sin ajustar y se colocó en cajas de plástico con tapa, las que se dispusieron en la germinadora programada con los siguientes parámetros: 12 horas luz, 12 oscuridad, $25^{\circ}$ C. El riego se realizó con agua destilada con ayuda de una pizeta y fue diariamente, para evitar que se seque el papel toalla, durante el tiempo que duró la prueba.

Las semillas parcialmente escarificadas y desinfectadas se sembraron en bandejas almacigueras sobre sustrato compuesto por arena, musgo y fibra de coco, colocando una semilla en cada orificio, a una profundidad de $5 \mathrm{~cm}$, y se llevaron al tinglado, identificando cada accesión con un rótulo, donde también se colocó el número de semillas y fecha de siembra. 
El riego se realizó en forma interdiaria, empleando una regadera y agua de uso doméstico. La temperatura del medio ambiente, fue de $16^{\circ} \mathrm{C}$ como mínimo y $23^{\circ} \mathrm{C}$ como máximo.

Las evaluaciones se realizaron a los 12 días después de la siembra, hasta los 30 días (germinadora) y hasta 45 días (tinglado), que concluyó la prueba, evaluando la viabilidad de las semillas, en base al número de semillas germinadas en ambos sustratos y teniendo en cuenta el tipo de escarificación, así como el contenido de humedad de la semilla con la que fue conservada.

\section{RESULTADOS Y DISCUSIÓN}

En la prueba de viabilidad, el $57 \%$ de accesiones evaluadas presentaron un rango de viabilidad óptimo de $80-100 \%$, en ambos métodos empleados. Los valores mínimos de $5 \%$ (tinglado) y $21 \%$ (germinadora), posiblemente este influenciado por la presencia de aceite en las semillas.

Tipo de sustrato utilizado. Los resultados obtenidos, nos indican que para la evaluación de la viabilidad de las semillas de sacha inchi, conservadas en cámara fría, se pueden utilizar cualquiera de los métodos empleados= La viabilidad de las semillas disminuye con el tiempo de almacenamiento (Álvares et al., 2020).

Escarificación. El tipo de escarificado influyó en la velocidad de germinación, las semillas escarificadas totalmente iniciaron el proceso de germinación a los 12 días, mientras que las semillas parcialmente escarificadas emergieron a los 20 días. De las 27 accesiones parcialmente escarificadas, seis de ellas presentaron porcentaje de germinación entre 80 $100 \%$ ), al igual que ocho accesiones con semillas completamente escarificadas.

Contenido de humedad de la semilla. El contenido de humedad de las semillas no influyó en el porcentaje de viabilidad de las semillas. La accesión con 4,0\% de contenido de humedad presentó $80 \%$ de viabilidad, en condiciones de tinglado, y la accesión con 7,5\%, presentó $85 \%$ de viabilidad en germinadora.
En base a los resultados obtenidos, en la Tabla 1., se confirma que las semillas de sacha inchi, tienen comportamiento ortodoxo, porque resisten a la desecación y se pueden mantener a bajas temperaturas, en recipientes herméticos, conservando su viabilidad (Kameswara, et al., 2007). Sin embargo, hay que evaluar el tiempo de longevidad, según (Mestanza, 2011), la semilla será más longeva cuando se disminuye su metabolismo. Asimismo, (Copete et al., 2018), afirma que las semillas ortodoxas, superan la latencia inicial, presentando mayor germinación antes del proceso de conservación, latencia inducida. (Vargas, 2009), reportó el mayor porcentaje de germinación hasta el $5^{\circ}$ mes de almacenamiento en cámara fría y condiciones naturales.

En relación al tiempo que duró la prueba de germinación en sacha inchi se establece que es entre 12 a 20 días para semillas completamente escarificadas y bajo condiciones controladas de luz y temperatura y de 20 a 45 días, para semillas parcialmente escarificadas y puestas al medio ambiente. Torres (2012), evaluó el efecto de ablandamiento y escarificación de semillas de sacha inchi, para acelerar el proceso de germinación y determinó 60 días. Asimismo, Vargas (2009), determinó que la viabilidad de semillas de sacha inchi fue aceptable hasta el quinto mes de almacenamiento. Pero cada especie tiene su propio proceso, por ejemplo Faife \& Toledo (2007) determinaron el proceso que dura la germinación en semillas de Melocaptus guitartii, conservadas por 6 y 12 meses a medio ambiente.

Las semillas de sacha inchi fueron conservadas durante ocho años en cámara fría en sobres de poliéster laminado, con contenidos de humedad que variaron entre 4,0\% a 7, 5\%. (Ruiz et al., 2017), refiere que semillas de Moringa oleifera $\mathrm{L}$, conservaron su calidad fisiológica, almacenadas en frío en envases de aluminio por 360 días. La calidad fisiológica es la capacidad de la semilla para germinar y producir una nueva planta, es decir que la semilla esté viable. 
Tabla 1. Porcentaje de Viabilidad en semillas de sancha inchi, en dos sustratos diferentes.

\begin{tabular}{|c|c|c|c|c|}
\hline Código Nacional & Identificación & $\begin{array}{c}\text { Cont. de Hd. } \\
(\%)\end{array}$ & $\begin{array}{c}\text { Germinación en papel } \\
(\%)(*)\end{array}$ & $\begin{array}{r}\text { Germinación en } \\
\text { sustrato }(\%)(* *) \\
\end{array}$ \\
\hline PER000394 & Shilcayo & 6,0 & 75,0 & 30,0 \\
\hline PER000395 & Pinto Recodo & 6,6 & 65,0 & 70,0 \\
\hline PER000396 & Cumbaza & 6,7 & 70,0 & 75,0 \\
\hline PER000397 & Barranquita & 6,5 & 21,0 & 15,0 \\
\hline PER000398 & Habana & 6,5 & 55,5 & 73,6 \\
\hline PER000399 & Tamishiyacu & 6,1 & 35,0 & 30,0 \\
\hline PER000401 & Caballococha & 6,7 & 72,7 & 50,0 \\
\hline PER000403 & Santa Clara (Alto) & 6,5 & 50,0 & 5,0 \\
\hline PER000404 & Estrecho (Bajo) & 6,7 & 75,0 & 65,0 \\
\hline PER000405 & Chirapa & 6,5 & 78,9 & 50,0 \\
\hline PER000406 & Muyuy & 6,7 & 80,0 & 75,0 \\
\hline PER000407 & Pacaya & 6,7 & 71,4 & 75,0 \\
\hline PER000408 & Rio Tigre & 4,0 & 47,3 & 80,0 \\
\hline PER000409 & Rio Mamon & 6,2 & 63,1 & 90,0 \\
\hline PER000413 & Tambo Yaguas & 6,2 & 40,0 & 90,0 \\
\hline PER000415 & Rio Samiria & 5,8 & 61,9 & 75,0 \\
\hline PER000416 & Tabatinga & 6,6 & 40,0 & 30,0 \\
\hline PER000417 & Chazuta & 6,7 & 85,0 & 94,7 \\
\hline PER000418 & Saposoa & 6,7 & 70,0 & 15,0 \\
\hline PER000419 & Moyobamba & 4,8 & 85,0 & 65,0 \\
\hline PER000420 & Por Identificar & 6,1 & 95,0 & 65,0 \\
\hline PER000421 & Rio Palmira & 6,6 & 77,7 & 85,0 \\
\hline PER000422 & Alto Shamboyacu & 5,4 & 85,7 & 57,8 \\
\hline PER000424 & $\mathrm{Si}-1$ & 5,4 & 35,0 & 30,0 \\
\hline PER017597 & Rio Yarapa & 5,9 & 100,0 & 70,0 \\
\hline PER018030 & Lamas & 7,5 & 85,0 & 72,2 \\
\hline PER018028 & Jeberos & 5,8 & 95,0 & 100,0 \\
\hline
\end{tabular}

(*) Emergieron a los 12 días $\quad$ (**) Emergieron los 20 días

De igual modo, (Ortiz, 2004), menciona que el almacenamiento de semillas en cámara fría y en envases herméticos sellados, prolongaron por un año la calidad fisiológica de semillas de soya, que es una oleaginosa como el sacha inchi. Otras especies también muestran buen comportamiento y conservan mejor la calidad fisiológica, cuando son almacenadas en refrigerador, como semillas de Berenjena Solanum melongena $\mathrm{L}$. en las que se determinó el contenido de humedad, germinación y vigor por un período de 11 meses (Aramendiz, et al., 2007).

Kameswara et al. (2007) señala que las semillas deben tener una viabilidad alta al inicio y durante el almacenamiento, para asegurar la producción de plantas cuando se siembre en campo. Cabe resaltar que, para la conservación de semillas, el rango de viabilidad aceptable es de $80-100 \%$. De igual manera el fotoperiodo también es importante en el proceso de germinación. En el experimento las semillas de sacha inchi fueron expuestas a un fotoperiodo de 12 horas luz, 12 oscuridad y temperatura de $25^{\circ} \mathrm{C}$. Ciertas especies requieren condiciones óptimas de luz y de oscuridad, además de temperatura. Tal es el caso para Aster pyrenaeus, cuyas condiciones óptimas para germinación fueron de 12 horas luz, 12 oscuridad y temperatura media de $20^{\circ} \mathrm{C}$ (Torrico et al., 2011).

La viabilidad en sacha inchi, depende también de la composición química de las semillas, ya que siendo del mismo lote de producción (año 2011), algunas presentaron valores de $21 \%$ a $35 \%$ (germinadora) y $5 \%$ a $35 \%$ (tinglado). Posiblemente se debe al alto contenido de aceite, presente en las accesiones. Acosta et al. (2019) refiere que las semillas oleaginosas presentan características especiales de germinación que regulan su propagación para acelerar la germinación de sacha inchi y emplearon giberelinas. La Rosa \& Quijada (2013), mencionan que sacha inchi se encuentra en estado silvestre. Sin embargo, no se conoce su manejo agronómico en condiciones 
controladas, variando la composición química de sus semillas.

En relación con los sustratos empleados, sacha inchi presentó una buena germinación en ambos sustratos, como se puede observar en la accesión PER018028, que presentó valores de $95 \%$ (papel) y $100 \%$ (arena, musgo y fibra de coco). Sin embargo, La Rosa \& Quijada (2013), encontraron alto porcentaje de germinación empleando como sustrato musgo fino, y refiere que este sustrato le proporciona el adecuado drenaje, aireación y retención de agua a las semillas para su germinación.

En relación a la escarificación de las semillas no influyó significativamente en el porcentaje de germinación, pero si en la velocidad de germinación, pues las semillas escarificadas completamente y bajo condiciones controladas de luz y temperatura, iniciaron el proceso de germinación a los 12 días, mientras que las semillas escarificadas parcialmente y sembradas en la mezcla de arena, musgo y fibra de coco y ubicadas en el tinglado, el proceso de germinación fue lento, iniciando a los 20 días, lo cual se debió a que la testa de las semillas de sacha inchi es gruesa, e impide el flujo necesario de agua y oxígeno para la germinación. La presencia de la testa y la permeabilidad para la absorción del agua en la germinación de sacha inchi, influye directamente, factor que ha sido poco estudiado (La Rosa \& Quijada, 2013).

Por otro lado, Torrico et al. (2011), refiere que la estratificación en frío aumentó la velocidad de germinación a $20^{\circ} \mathrm{C}$, al igual que a $15^{\circ} \mathrm{C}$ en semillas de Aster pyrenaeus y no hubo diferencias significativas en la viabilidad de los frutos. Asimismo, Duarte et al. (2014), evaluaron el efecto de la testa en la germinación de semillas Handroanthus heptaphyllus, almacenadas en cámara fría a $4^{\circ} \mathrm{C}$, durante $1,6,12$ y 24 meses, que descendió de $100 \%$, hasta hacerse nulo a los 24 meses, pero al remover la testa el porcentaje de viabilidad aumento, demostrando la influencia de la testa y los bajos valores obtenidos. La Rosa \& Quijada (2013), determinaron valores bajos de porcentaje de germinación e índice de velocidad de germinación empleando semillas con testa, posiblemente debido al sustrato empelado que no le proporcionó un drenaje adecuado, lo cual disminuyó el oxígeno e inhibición de la germinación, además la alta humedad provocó desarrollo de hongos. Coronado (2018), evaluó las características morfológicas y niveles de infección fúngica en la germinación de semillas de sacha inchi, la calidad morfológica y sanitaria son muy importantes en la industria semillera.

En general los resultados obtenidos empleando ambos sustratos, nos indican que se tendrá que seguir evaluado para establecer el tiempo de conservaciónde las semillas de sacha inchi, de las accesiones que obtuvieron entre $80-100 \%$ de viabilidad, el resto de material se tiene que renovar, solicitando nueva semilla para su procesamiento y conservación.

\section{CONCLUSIONES}

En base a los resultados obtenidos, se concluye que las semillas de sacha inchi son ortodoxas y pueden ser desecadas para su almacenamiento y conservación a bajas temperaturas. Sin embargo, hay que establecer el periodo de conservación. Algunas accesiones y/o ecotipos presentan mayor cantidad de aceite en su composición química, que influye en el proceso de deterioro y baja viabilidad. Se debe seguir evaluando el porcentaje de viabilidad de las accesiones que aún presentan un rango aceptable de germinación y determinar hasta qué tiempo se puede conservar en cámara fría. Mientras que, la velocidad de germinación en las semillas de sacha inchi, está influenciado por la presencia de la testa. Las semillas completamente, escarificadas germinan más rápido que las parcialmente escarificadas.

\section{Conflictos de intereses}

Los autores firmantes del presente trabajo de investigación declaran no tener ningún potencial conflicto de interés personal o económico con otras personas $\mathrm{u}$ organizaciones que puedan influir indebidamente con el presente manuscrito.

\section{Contribuciones de los autores}

Preparación y ejecución: FC; AR; Desarrollo de la metodología: FC; AR; Concepción y diseño: FC; AR; Edición del artículo: FC; AR; Supervisión del estudio: FC; AR 


\section{REFERENCIAS}

- Acosta, J., Lorente, G., Pérez, M., González, M., Michelena, G., García, R., Cortés, R., \& González, J. (2019). La germinación de semillas de Sacha Inchi regulada por Giberelinas derivadas de la caña de azúcar: primera experiencia cubana. ICIDCA, 53(1),54-57.

https://www.researchgate.net/publication/3399166 52

- Alayon, A., \& Echeverri, I. (2016). Sacha Inchi (Plukenetia volubilis linneo): ¿una experiencia ancestral desaprovechada? Evidencias clínicas asociadas a su consumo. Revista chilena de nutrición, 43(2), $167-$ 171. https://dx.doi.org/10.4067/S071775182016000200009 .

- Alcívar, J. (2014). Estudio de factibilidad para la industrialización de un aceite de alta calidad nutricional y medicinal extraido de sacha inchi. (Tésis Universidad Católica Santiago de Guayaquil. Facultad de Especialidades empresariales, Guayaquil. Recuperado en: repositorio.ucsg.edu.ec/bitstream/3317/2221/1/TUCSG-PRE-ESP-CFI-60.pdf

- Álvarez, O., Pérez-Reyes, C., \& Bonilla-Vichot, M. (2020). Evaluación de la viabilidad en semillas de Pinus tropicales Morelet con diferente tiempo de almacenamiento. Avances, 22(1), 97-109.

- Antoniolli, F. \& Arfini, F. (2013). Sacha inchi. Investigación sobre las condiciones para el reconocimiento d ela indicación geográfica en el Perú. Centro de Investigación y Desarrollo -CIED. Fundación Terre des home Italia. Disponible en https://www.researchgate.net/publication/3038514 51_Esta publicación no hubiera sido posible sin el apoyo moral y técnico de Belén Garví Medina, Elisa Dallatana, Mauro Morbello, Barbara Lohnmeier.

- Aramendiz, H., Cardona, C., Jarma, A., Robles, J., \& Montalván, R. (2007). Efectos del almacenamiento en la calidad fisiológica de la semilla de berenjena (Solanum melongena L.). Agronomía Colombiana, 25(1):104-112. https://www.redalyc.org/articulo.oa?id=1803/1803 16240012

- Brack, A. (1999). Diccionario Enciclopédico de Plantas Medicinales Útiles del Perú. Cuzco: Centro
De Estudios Regionales Andinos Bartolomé de las Casas.

- Brack, A. (2003). Perú: diez mil años de domesticación. Lima, Perú. Editorial Bruño.

- Brack, A., \& Bravo, F. (2005). Perú Legado Milenario. Lima, Perú. Universidad San Martín de Porres.

- Copete, M. Á., Herranz, R., Ferrandis, P., \& Herranz, J. (2018). Inducción de latencia en semillas desecadas de Scorzonera reverchonii (Compositae) conservadas a baja temperatura. Bosque, 39 (1), 137-143. doi: 10.4067/S071792002018000100137

- Coronado, N. (2018). Evaluación de características morfológicas y niveles de infección fúngica natural en la germinación de semillas de sacha inchi (Plukenetia volubilis) provenientes de diferentes regiones de Colombia (Tesis, Universidad de Santander, Facultad de Ciencias exactas, naturales y agropecuarias microbiología industrial Bucaramanga, Colombia). Recuperado de https://cutt.ly/MaWaSv2

- Doria, J. (2010). Revisión bibliográfica Generalidades sobre las semillas: su producción, conservación y almacenamiento. Cultivos Tropicales, 31(1), 74-85. Recuperado de http://exa.unne.edu.ar/biologia/fisiologia.vegetal/g eneralidadessobrelassemillas.pdf

- Dosert N., Roque, J., Brokamp, G., Echevarria, A., Torre, M., \& Weigened, M. (2009). Factsnat: Datos Botánicos de Sacha Inchi Plukenetia volubilis L.

- https://www.researchgate.net/publication/4317881 7

- Duarte E., Avico E., Sansberro P., \& Luna C.E. (2014). Efecto de la testa sobre la germinación de semillas de handroanthus heptaphyllus tras distintos tiempos de almacenamiento. Revista Ciencias Agronómicas, XXIV - 29 / 29-35. https://ri.conicet.gov.ar/handle/11336/2841

- Faife, M., \& Toledo, P. (2007). Viabilidad de semillas de Melocactus guitartii León conservadas por seis y doce meses. Centro Agrícola, 34(1), 4750.

http://cagricola.uclv.edu.cu/descargas/pdf/V34Numero_1/cag061071526.pdf

- Flores, D. (2010). Uso histórico del sacha inchi. Plukenetia volubilis. L. Peru Proyecto Perú Biodiverso. 
https://repositorio.promperu.gob.pe/bitstream/han dle/123456789/1371/Uso_historico_sacha_inchi_ 2010_keyword_principal.pdf?sequence $=1$

- García, L. (2015). Determinación de la viabilidad de semillas de sacha inchi (Plukenetia volubilis 1.) en vivero, accesión pinto recodo, bajo seis formas de conservación. (Tesis de Licenciatura, Universidad Nacional de San Martín Tarapoto, Facultad de Ciencias Agrarias. Tarapoto, Perú). Disponible en http://repositorio.unsm.edu.pe/handle/11458/627

- García-López, J., Ruíz Torres,A., Lira-Saldivar, R., Vera-Reyes, I., \& Méndez-Argüello, B. (2016). Técnicas Para Evaluar Germinación, Vigor y Calidad Fisiológica de Semillas Sometidas a Dosis de Nanopartículas. Capítulo de libro. Universidad Autónoma Agraria Antonio Narro, Saltillo, México. Disponible en http://ciqa.repositorioinstitucional.mx/jspui/hadle/ $1025 / 334$

- Hernández-Coronado.A., Rodriguez, A., Candre A., Pinto, I., Miraña, E., \& Bernilla, A. (2018). Estudio de Viabilidad y conservación de Semillas de Cinco especies Vegetales Amazónicas como base para la creación de un Banco de germoplasma en el Municipio de Leticia, Amazonas, Colombia. Folia Amazónica. Revista del Instituto de la Amazonía Peruana, 27(1), 9-23. http://revistas.iiap.org.pe/index.php/foliaamazonic a/article/view/435/513

- Kameswara, R., Hanson, J., Dulloo,E., Ghosh, K., Nowell, D., \& Larinde, M. (2007). Manual ara el Manejo de Semillas en Bancos de Germoplasma.Bioversity International. Roma, Italia.

- La Rosa R., \& Quijada, J. (2013). Germinación del sacha inchi, Plukenetia volubilis 1. (mcbride, 1951) (malpighiales, euphorbiaceae) bajo cuatro diferentes condiciones. The Biologist (Lima),11(1),11-14.

- Lazos-Monterrosa, FA., Orantes-García, C., Farrera-Sarmiento, O., Verdugo-Valdez, AG., Sánchez-Cortés, MS., \& Ruíz-Meza, LE. (2015). Evaluación de la viabilidad y germinación de tempisque [Sideroxylon capiri (A.DC.) Pittier Sapotaceae]. Revista Internacional de Botánica Experimental, 84 (1).
- Mancipe, C., Calderón, M., \& Pérez, L. (2018). Evaluación de viabilidad de semillas de 17 especies tropicales altoandinas por la prueba de germinación y la prueba de tetrazolio. Caldasia, 40 (2),366-382. https://revistas.unal.edu.co/index.php/cal/article/vi ew/68251/70378

- Mestanza, H. (2011). Viabilidad de la semilla de piñón (Jatropha curcas 1.) mediante métodos de almacenamiento natural y artificial en la región San Martín (Tesis de Licenciatura, Universidad Nacional de San Martín Tarapoto, facultad de Ciencias Agrarias. Tarapoto, Perú). http://repositorio.unsm.edu.pe/bitstream/handle/11 458/587/TFCA_125.pdf?sequence $=1 \&$ isAllowed $=$ $\mathrm{y}$

- Navarro, M., \& Lezcano, J.C. (2008). Efecto del método de secado en la longevidad y calidad de las semillas de Bauhinia purpurea.: II. Almacenamiento en cámara fría. Pastos y Forrajes, 31(1), 1. http://scielo.sld.cu/scielo.php?script=sci_arttext\& pid=S086403942008000100005\&lng=es\&tlng=es.

- Ortiz, R., \& Ponce, M. (2004). Evaluación de métodos de almacenaje de semilla de soya (Glycine max. (L.) Merrill) en condiciones de bajos insumos. Cultivos Tropicales, 25(3),49-58. https://www.redalyc.org/articulo.oa?id=1932/1932 17916006.

- Ruiz, A., Aramenndiz, H., \& Cardona, C. (2017). Efecto del almacenamiento en la calidad fisiológica de semilla de moringa (Moringa oleifera Lam.). Revista U.D.C.A. Act. \& Div. Cient, 20(1),79-89. https://revistas.udca.edu.co/index.php/ruadc/articl e/view/65

- Sánchez, J., Parra, A., Silva M., \& Pedroza, D. (2011). Efecto de la temperatura y tiempo de almacenamiento sobre la viabilidad en semillas de zámota (Coursetia glandulosa, gray). Revista Biotecnia.

- Torres, M. (2012). Efecto del ablandamiento y escarificado en la germinación de semillas de sacha inchi (Plukenetia volubilis), ecotipo Pinro Recodo, en Pucallpa (Tesis de Licenciatura, Universidad Nacional de Ucayali, Facultad de Ciencias Agropecuarias. Pucallpa, Perú). https://alicia.concytec.gob.pe/vufind/Record/RUN U_6018dc19e7f4004a71415aa7d78a0922 
- Torrico, A., Jiménez-Alfaro, B., \& Pascual, E. (2011). Implicaciones de la viabilidad y germinación de semillas en la conservación de Aster pyrenaeus desf. ex -dC.(Asteraceae). Andorra, 147-154. https://cutt.ly/FaWInd9

- Vargas, G. (2009). Determinación de la viabilidad de semilla de sacha inchi (Plukenetia volubilis) bajo condiciones de almacenamiento en cámara fría y condiciones normales en Tarapoto (Tesis de Licenciatura, Universidad Nacional de San Martín Tarapoto, Facultad de Ciencias Agrarias. Tarapoto, Perú).

http://repositorio.unsm.edu.pe/bitstream/handle/11 458/765/TP-

FAGRO_0405.pdf?sequence=1\&isAllowed=y 